\title{
Focal dystonia of the jaw and the differential diagnosis of unilateral jaw and masticatory spasm
}

\author{
PD THOMPSON, JA OBESO,* G DELGADO, $\dagger$ J GALLEGO, $\dagger$ CD MARSDEN
}

\author{
From the University Department of Neurology, Institute of Psychiatry \& King's College Hospital Medical \\ School, London UK, the Movement Disorders Unit, Department of Neurology, Clinica Universitaria, University \\ of Navarra, ${ }^{*}$ and the Neurology Service, Hospital Provincial de Navarra, $\dagger$ Pamplona, Spain.
}

SUMMARY The clinical features, differential diagnosis and treatment of unilateral spasms of the jaw and masticatory muscles are discussed and illustrated by eight cases of unilateral jaw spasms of various aetiologies. These include focal dystonia of the jaw, hemimasticatory spasm with and without facial hemiatrophy, paroxysmal events in multiple sclerosis and tetany. Attention is particularly drawn to four cases of unilateral dystonia of the jaw which has not been described before.

In a discussion of the "masticatory spasm of Romberg", Gowers described several conditions which were associated with tonic or clonic jaw spasms. ${ }^{1}$ He referred to tetanus, trauma, tetany, facial pain, hysteria, pontine lesions and cold exposure as causes of tonic spasm or trismus; he listed convulsions, rigors, paralysis agitans, facial pain, and chorea among the causes of clonic jaw spasm. Such spasms must be distinguished from the involuntary movements of the jaw which occur in a number of other conditions. These include generalised and cranial dystonia, ${ }^{2}$ acute dystonic reactions to neuroleptic drugs, ${ }^{3}$ tardive dyskinesia, ${ }^{3}$ tardive dystonia, ${ }^{4}$ dyskinesias induced by levodopa and related drugs, and post-encephalitic Parkinsonism. ${ }^{5}$ Trismus and bruxism or teeth grinding may also be encountered in pseudobulbar palsies due to cerebrovascular disease. ${ }^{6}$ Bruxism is also considered to be a manifestation of anxiety. ${ }^{7}$ In addition, a variety of complicated masticatory movements may be seen in epileptic automatisms and in response to oral or facial stimulation in patients with severe damage of the cerebral hemispheres or brainstem. ${ }^{8}$ Finally, masticatory spasm may occur in peripheral neuromuscular disorders that result in muscle spasms or cramp, and is a prominent late feature of the clinical syndrome of progressive muscle spasm, alopecia and diarrhoea. ${ }^{9}$

In most of these disorders, the jaw spasms are bilateral and occur as one facet of a generalised disorder of

\footnotetext{
Address for reprint requests: Professor CD Marsden, Department of Neurology, Institute of Psychiatry, De Crespigny Park, London SE5 8AF, UK.
}

Received 1 October 1985. Accepted 18 November 1985 movement. In contrast isolated unilateral jaw spasms are distinctly unusual. Apart from a number of case reports of masticatory spasm, most occurring in association with facial hemiatrophy, ${ }^{10-12}$ few references to unilateral jaw spasms are to be found in the literature.

The purpose of this report is to describe the clinical features of eight patients with unilateral jaw spasms of differing aetiologies causing unilateral deviation of the jaw and to discuss the clinical and electromyographic differential diagnosis of this condition.

\section{Methods}

Four of the eight patients described in this report were seen in the Department of Neurology at the Maudsley Hospital, three in the Department of Neurology, University of Navarra Medical School, Pamplona, Spain and one in the Department of Neurology, Sir Charles Gairdner Hospital, Perth. The patients were all females and their ages ranged from 24 to 60 years. Detailed electromyographic recordings and electrophysiological studies of the facial and masticatory muscles were performed in selected patients. The EMG recordings were made using silver/silver chloride surface electrodes, placed over the muscles of interest, and concentric needle electrodes were used to sample the motor unit discharge pattern in the masseter, pterygoid and orbicularis oris muscles. The EMG signals were amplified (Devices 3120 amplifier; band width from $80 \mathrm{~Hz}$ to $2.5 \mathrm{KHz},-3 \mathrm{~dB}$, for both surface and needle recordings) and recorded on a PDP 12 computer (sampling rate $40 \mathrm{KHz}$ ). In patients 1,3 and 5 the EMG signals were recorded directly on magnetic tape (Racal store 7DS) for later analysis. In patients 4, 6, and 7 the EMG recordings were made using a Medelec MS6 system. Exteroceptive suppression in the masseter muscles during masseter contraction (the masseter silent period), and the blink reflex and its recovery cycle were measured according 

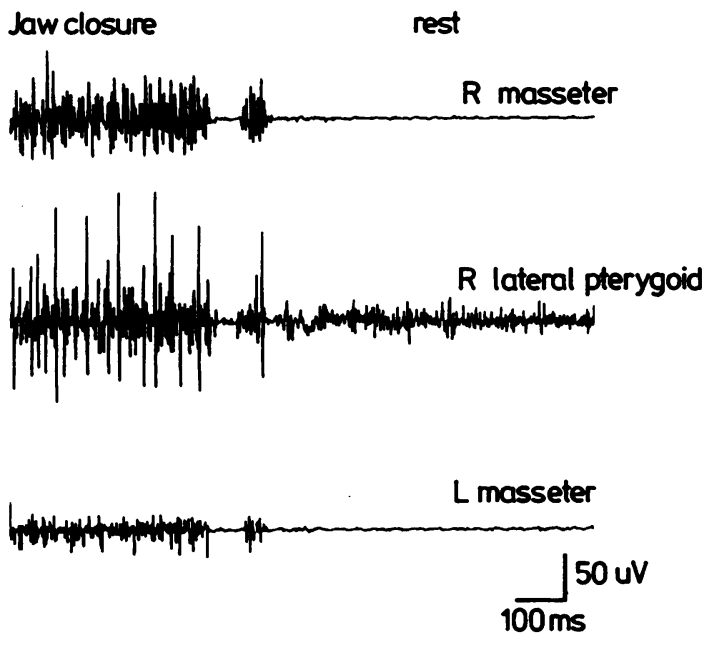

Fig 1 EMG recordings (concentric needle electrodes) from the masticatory muscles in unilateral jaw dystonia (case 1). In the position of maximal jaw deviation, activity was recorded in the contralateral lateral pterygoid, while the masseter muscles were silent (right half of record). If the patient forcibly returned her jaw to the midline, and held it in that position by clenching her teeth, the activity continued in the pterygoid and was co-contracting with the masseter (left half of record).

to the methods described by Berardelli et al. ${ }^{13}$ The latency of the masseter reflex was measured following mechanical stimulation of the jaw with a tendon hammer.

\section{Case reports}

Case 1 Isolated unilateral jaw dystonia

A 24-year-old woman first became aware of abnormal jaw movements at age 14 years when her jaw began to deviate to the left. This would persist for several weeks at a time before resolving spontaneously. Over the following years the jaw deviation recurred intermittently, often lasting for several months at a time before returning to normal. Each episode varied in duration and the extent of lateral jaw deviation. During them she found it difficult to chew or talk. At times she experienced pain and tingling in the region of the left mandible. The disfigurement produced by the jaw deviation caused considerable embarrassment. She underwent several treatments including a variety of dental procedures and had been prescribed a number of drugs, without benefit. There was no significant family history and she took no regular medications. Examination during an attack revealed sustained deviation of the jaw to the left, giving the face an asymmetric appearance. When free of symptoms the examination was normal. A number of investigations including high resolution computed tomography of the brain and posterior fossa, cerebrospinal fluid examination and visual and auditory evoked responses were normal. EMG recordings of the motor unit potentials in the jaw muscles indicated that sustained activity in the right lateral pterygoid was responsible for the (contralateral) jaw deviation (fig 1). The morphology of the motor units appeared normal. In the deviated position activity was recorded only from the pterygoid muscle. However, when the jaw returned to the mid-line (which the patient was able to achieve with some effort), co-contraction of the masseter and pterygoid muscles on the right was present (fig 1). The masseter reflex latencies were normal $(9 \mathrm{~ms} ; R=L)$ as were the latencies of the early and late components of the blink reflex $(R 1=10 \mathrm{~ms}, \mathrm{R} 2=40 \mathrm{~ms}$, $R=L$ ). The recovery cycle of the blink reflex was normal.

Benzhexol, in daily doses of 20 to $30 \mathrm{mg}$, was initially effective in almost completely abolishing the prolonged periods of jaw deviation, although short episodes have since reappeared.

\section{Case 2 Unilateral jaw dystonia preceded by temporary torticollis}

A 37-year-old woman developed torticollis with the chin deviated to the left, at age 30 years. This became increasingly troublesome over a period of 2 years before resolving after vigorous neck manipulation. A short time later she developed involuntary opening of her mouth and deviation of the jaw to the left. She found chewing difficult and would often bite her tongue. These movements were painful and the source of considerable embarrassment on account of the

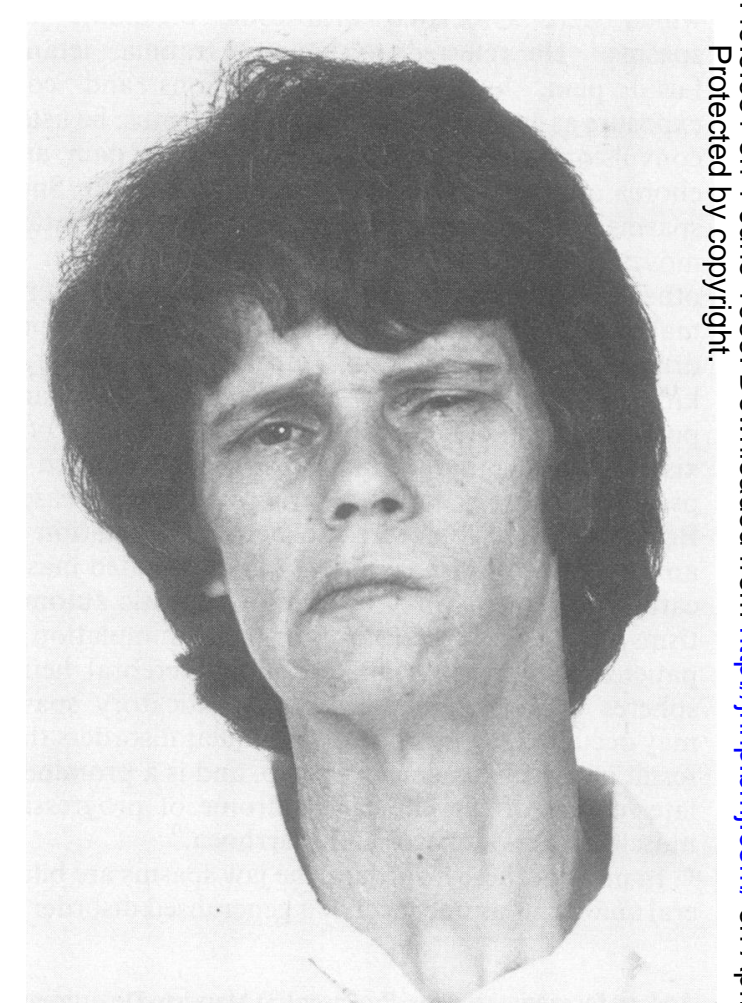

Fig 2 Deviation of the jaw to the right, producing an asymmetric face and giving the impression of facial weakness in a patient with jaw dystonia and torticollis (case 3). 


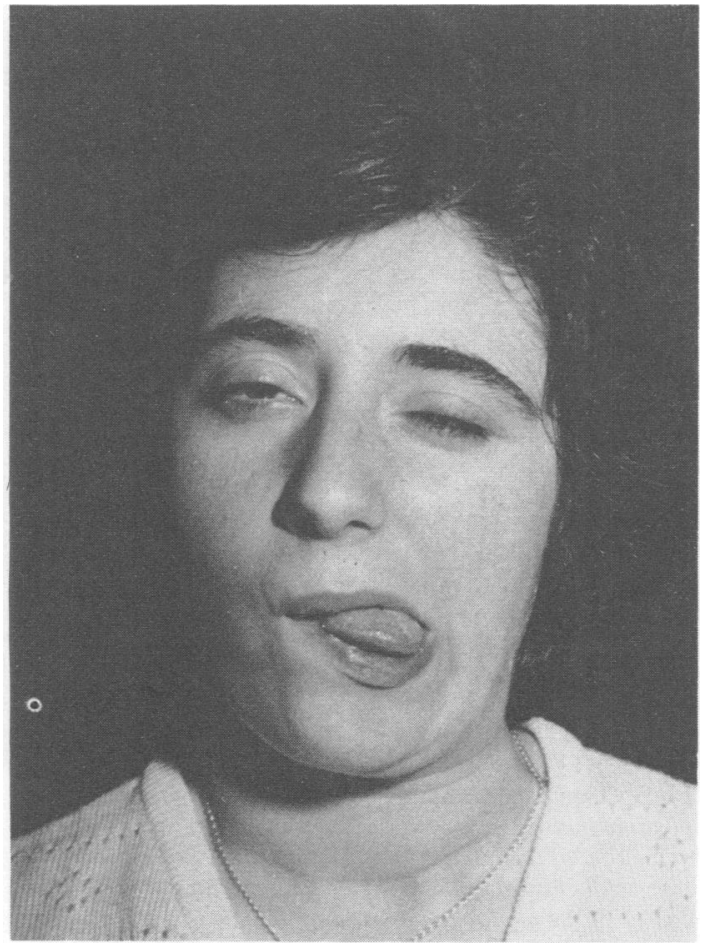

Fig 3 Jaw dystonia in a patient with cranial and segmental dystonia (case 4). Note the deviation of the jaw to the left, left sided blepharoclonus, drawing of the lips to the left and deviation of the protruded tongue to the left.

facial disfigurement they produced. Two years later she began to develop sustained spasms of jaw opening, particularly when eating. She was also aware of a numb sensation affecting the left side of the face. She had tried numerous therapies and drugs without benefit. There was a family history of retinitis pigmentosa. Examination revealed a pigmentary retinopathy with constriction of the peripheral visual fields. Intermittent spasms of the jaw muscles produced jaw opening and/or deviation of the jaw to the left. Facial sensation, the corneal reflexes and the jaw jerk were normal, as was the remainder of the examination.

Benzhexol, in a dose of 15 to $20 \mathrm{mg}$ per day, reduced but did not abolish the involuntary movements.

\section{Case 3 Unilateral jaw dystonia with torticollis}

A 42-year-old woman developed painful movements of the jaw with deviation of the jaw to the right following a dental extraction, at age 35 years. The movements and the degree of jaw deviation fluctuated during the 7 year history but never disappeared completely. The movements interfered with chewing and she often bit her tongue. A variety of dental procedures were performed without benefit, including extraction of her remaining teeth. She had not been aware of any other involuntary movements. There was a past history of depression but she had not received any drugs before the onset of the jaw movements. There was no significant family history. Examination revealed sustained deviation of the jaw to the right, producing an asymmetric face which gave the impression of facial weakness (fig 2). Facial movements and sensation were normal. There was intermittent torticollis to the right. The remainder of the examination was normal. Investigations including computed tomography of the brain were normal.

EMG recordings revealed normal motor unit morphology and patterns of discharge in the masseter muscles. The masseter reflex latencies were normal ( $8.5 \mathrm{~ms}$ bilaterally) as was the masseter silent period on both sides. The blink reflex latencies were also normal $(R 1=12 \mathrm{~ms}$ and $R 2=29 \mathrm{~ms}$; $R=L$ ) but the recovery cycle of the blink reflex following a conditioning stimulus was enhanced in a manner similar to that described in patients with oromandibular dystonia and blepharospasm. ${ }^{13}$

Treatment with benzhexol in daily doses of between 25 and $35 \mathrm{mg}$ lessened the severity of the jaw movements. However, she became depressed and the treatment was gradually withdrawn. The movements worsened but later improved with amitriptyline $100 \mathrm{mg}$ per day.

\section{Case 4 Unilateral jaw dystonia with cranial and segmental} dystonia

A 22-year-old female developed deviation of the jaw to the left and intermittent closure of the left eye, associated with a tingling sensation of the left forehead. These symptoms resolved spontaneously over a period of 3 weeks but recurred 4 weeks later. She has continued to experience attacks, each lasting for weeks at a time, before settling, with symptom free intervals lasting several weeks. There was no significant past history and no history of drug ingestion. Examination during an attack revealed intermittent blepharoclonus of the left orbicularis oculi and sustained deviation of the jaw and lips to the left (fig 3). On protruding the tongue, it too deviated to the left. There was a torticollis with the chin turned to the left and dystonic inversion of the outstretched left arm. The remainder of the examination was normal. Investigations including computed tomography of the brain, vertebral arteriography and cerebrospinal fluid analysis were normal. Visual and somatosensory evoked responses were normal as were EMG recordings from the left masseter and blink reflex latencies.

Treatment with anticholinergics, carbamazepine, clonazepam, haloperidol and tetrabenazine did not provide any relief.

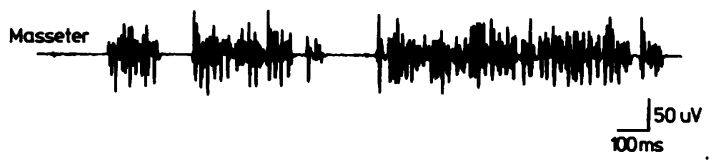

Fig 4 Electromyographic (concentric needle electrode) recording from masseter in hemimasticatory spasm with facial hemiatrophy (case 5) illustrating the sudden onset and offset of bursts of spontaneously discharging motor units. Note the variable burst duration lasting from 50 to $300 \mathrm{~ms}$ as illustrated in the first three bursts. The next brief discharge $(20 \mathrm{~ms})$ is followed by two longer bursts each approximately of $400 \mathrm{~ms}$ duration, punctuated by another short discharge. 


\section{Case 5 Unilateral hemimasticatory spasm with facial} hemiatrophy

A 38-year-old woman had been aware of abnormal pigmentation of the right side of her neck, lower face and jaw since childhood. During her teens she was aware of progressive atrophy of these regions and a series of skin biopsies revealed changes consistent with morphoea. At age 31 years she developed spasms of the muscle of the right jaw which caused it to close forcibly. Each spasm would begin with a series of small "warning" contractions which she felt as a tightening of the face, and last for a second or two. They were often precipitated by mouth closure or movement of the jaw while eating. Opening the jaw would interrupt the spasm. If a prolonged spasm developed it would last for several minutes. She had bitten her tongue on numerous occasions and at times the spasms woke her at night. They had been temporarily abolished by mandibular anaesthesia during the fitting of a dental plate. A masseter myotomy did not improve her symptoms but carbamazepine and phenytoin had greatly reduced the severity of attacks. She was also aware of spasm of the right lower lip which pulled the corner of her mouth down on that side for several seconds at a time. Examination revealed patchy pigmentation of the skin and atrophy of the subcutaneous tissue of the right side of the neck and face. The masseter was visibly hypertrophied and repetitive contractions of it could be seen and palpated during a spasm of jaw closure. The jaw jerk was present and there was no weakness of jaw opening or closure. Facial sensation was normal as were facial movements when there was no spasm of the lower facial muscles. Spasm and deviation of the lower lip down and to the right were present intermittently and could be induced by stretching the lip. The remainder of the neurological examination was normal.

EMG recordings from both masseter muscles revealed spontaneous activity in the right masseter while the left remained silent except during voluntary contraction. The spasms in the right masseter consisted of repetitive, spontaneous bursts of motor unit discharges, varying in duration from 20 to $400 \mathrm{~ms}$ (fig 4). Similar bursts were recorded in the right orbicularis oris muscle. The latency of the masseter reflex on the right $(11 \mathrm{~ms})$ was prolonged in comparison to that on the normal side $(7 \mathrm{~ms})$. Blink reflex latencies were normal $(R 1=10 \mathrm{~ms}, R 2=35 \mathrm{~ms} ; R=L)$ following stimulation of the right supraorbital nerve, as was the recovery cycle of the reflex following a conditioning stimulus. The terminal latency of the facial nerve to the orbicularis oris following stimulation anterior to the mastoid process was normal (3-4 ms). She continues to take carbamazepine and phenytoin.

Case 6 Hemimasticatory spasm without facial hemiatrophy A 60-year-old woman presented with a 3 year history of painful spasm of the left temporalis and masseter muscles, the details of which have been reported previously. ${ }^{12}$ The spasms were similar to those described in case 5, with repetitive brief contractions of the masseter and temporalis muscles. They occurred spontaneously and were induced by jaw closure but interrupted by jaw opening. There was no evidence of facial hemiatrophy and there was unilateral hypertrophy of the masseter and temporalis muscles involved. Numerous drugs were tried without benefit, and extensive investigation failed to reveal any underlying structural

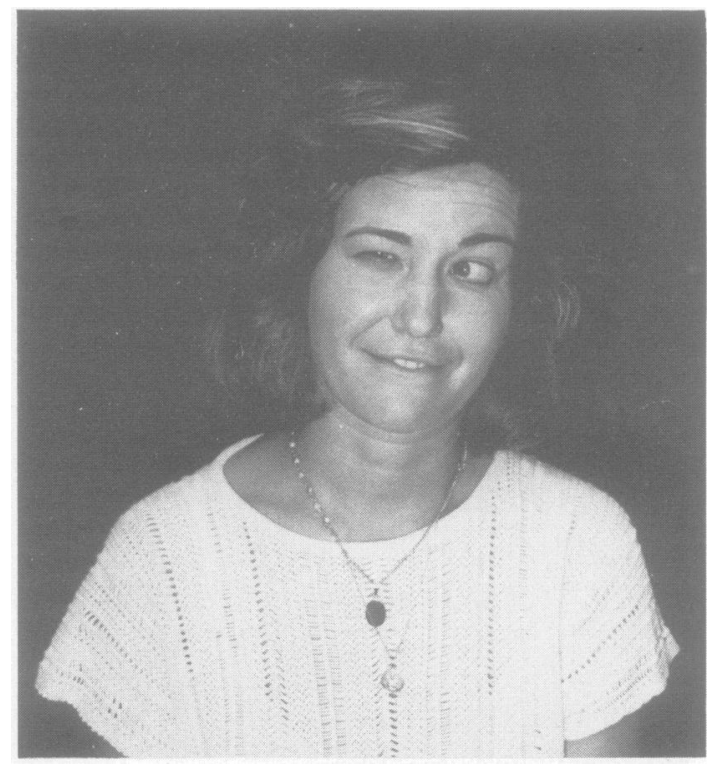

Fig 5 Complex facial and masticatory movements in possible multiple sclerosis (case 7). Note the spasm of adduction of the left eye, contraction of the frontalis on that side, with closure of the right eye and elevation of the right corner of the mouth and closure of the jaw which was deviated to the right.

abnormality of the brain. The jaw jerk was absent on the left and EMG recordings of the spontaneous activity were similar to those described in case 5 , with repetitive short bursts $(20-100 \mathrm{~ms})$ of motor units and longer spasms lasting up to $1 \mathrm{~s}$.

Case 7 Paroxysmal unilateral spasms of the jaw, associated with possible multiple sclerosis

A 28-year-old woman developed difficulty closing the right eye and deviation of the mouth to the left, which recovered over several weeks. Two months later she developed short attacks in which the mouth and jaw would deviate to the right and the right eye would close forcibly. These would last for 1 to 2 minutes and often recurred in bouts lasting several days. No precipitating factors were identified. There was no past history of significance and she took no regular medications. Examinations during an attack revealed that it would begin with closure of the right eye followed by elevation of the right corner of the mouth and closure and deviation of the jaw to the right, accompanied by spasms of the right orbicularis oculi, oris, mentalis, left frontalis and adduction of the left eye (fig 5). The remainder of the cranial nerves were normal. When walking the right foot would adopt a posture of plantar flexion and inversion which would last a minute or so and subside with rest. There was no muscle weakness but the deep tendon reflexes were exaggerated, especially in the legs. The superficial abdominal reflexes were absent and the plantar responses were flexor.

Investigations including computed tomography of the 


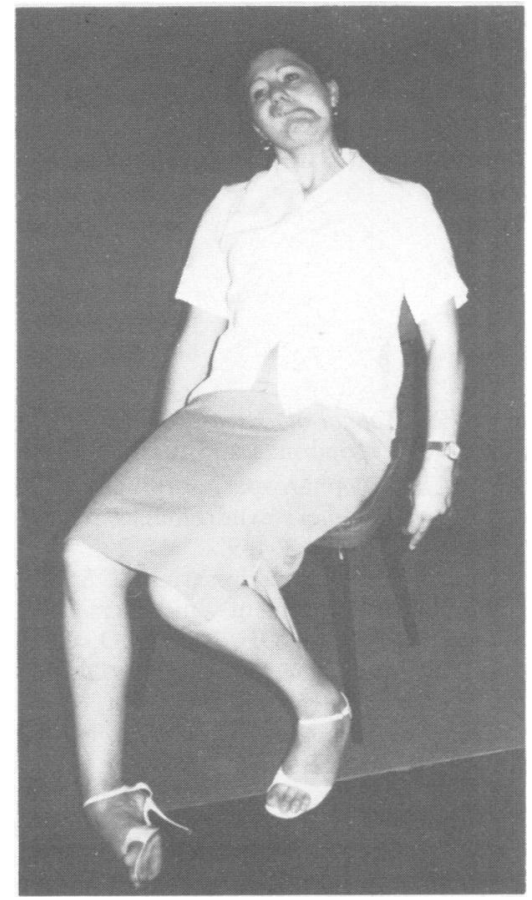

Fig 6 Spasms in tetany (case 8) involving the face, jaw and limbs. In addition to the bizarre distortion of the facial muscles and spasm of the jaw muscles, typical carpopedal spasm also is present.

brain, antinuclear factor, serum and urinary copper levels, visual and somatosensory evoked potentials and cerebrospinal fluid analysis were normal. EMG recordings from the orbicularis oculi and oris during an attack showed motor units of normal morphology.

Brief attacks have continued without any obvious precipitating factors.

\section{Case 8 Unilateral spasm of the masticatory and facial muscles in tetany}

A 32-year-old woman presented with episodic spasm of the jaw, face, neck and limb muscles. She was not aware of any precipitating factors or warning symptoms. Each attack lasted between 2 and 5 minutes and was followed by complete recovery. There were no associated symptoms. Her past and family history were unremarkable and she did not take any medications. A typical attack was observed to begin with hyperventilation and some 30 seconds later she would develop spasm of the facial and masticatory muscles producing a bizarre distortion of the face (fig 6). The neck muscles would then contract particularly on the left side, so that her head turned to the right. In addition she would develop carpopedal spasm with inversion and internal rotation of the feet. The injection of $1 \mathrm{~g}$ of calcium gluconate intravenously produced total relief of the symptoms in less than 1 minute. The EEG did not change during attacks and serum calcium levels were normal both during attacks and when free of symptoms.

\section{Discussion}

The differential diagnosis of causes of unilateral jaw spasms with or without involvement of the facial muscles is shown in the table. Of these conditions, mechanical and inflammatory disorders of the temporomandibular joint and oromandibular region will be evident on clinical examination. Tetanus may have to be borne in mind as cephalic tetanus has been described with unilateral facial weakness and trismus as presenting symptoms. ${ }^{14}$ Focal motor epilepsy will be distinguished by the brevity of attacks, the repetitive clonic nature of the jaw (and facial) contractions, spread of movements elsewhere and other ictal events. The remaining causes of unilateral jaw spasm with jaw deviation are relatively uncommon. Among these, focal dystonia of the jaw, presenting as an isolated involuntary movement with sustained lateral deviation of the jaw is the least well recognised cause of jaw spasms. In the cases of unilateral jaw dystonia described in this report, deviation of the jaw would persist for several days to weeks at a time before remitting, only to be followed by a further episode after a symptom free interval. In two patients (cases 3 and 4) the presence of more familiar dystonic postures at other sites (the neck and arm) confirmed that the jaw movements were part of a dystonic syndrome. In a further patient (case 2), jaw spasm was preceded by torticollis (which had resolved spontaneously) and was followed by the evolution of typical oromandibular dystonia, with spasms of jaw opening replacing the spasms of jaw deviation. Such changes in the nature of the involuntary jaw movements in oromandibular dystonia have previously been described $^{2}$ and illustrate the variable natural history of dystonia. In one other patient (case 1), jaw dystonia was the only abnormality, but this was similar in all aspects to the jaw spasms seen in the other three patients (cases 2, 3 and 4) who had evidence of dystonia elsewhere.

Investigation of these patients with jaw dystonia failed to reveal any underlying abnormality of the brain. Accordingly, they must be described as having idiopathic or primary dystonia. The morphology and discharge pattern of motor units recorded from the masseter, facial and the lateral pterygoid muscles (in

Table Differential diagnosis of abnormal unilateral involuntary movement of the jaw and face

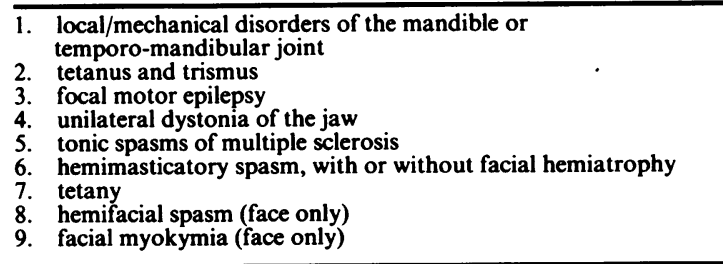


cases 1 and 3) were normal. In one patient (case 1) it was possible to record the continuous lateral pterygoid activity that was responsible for the jaw deviation in the "resting" position. When the patient attempted to straighten her jaw and clench her teeth, cocontraction of the masseter and pterygoid muscles was present. This example of jaw dystonia is similar to a further case cited by Gowers in whom fixed lateral deviation of the jaw was thought to be "... due to spasm in the pterygoids of one side...". 1 In one patient (case 3) detailed study of the blink and masseter reflexes disclosed an abnormality similar to that described in patients with oromandibular dystonia and blepharospasm ${ }^{13}$ further supporting the postulate that the jaw movements in this case were part of a restricted cranial dystonia. Treatment with anticholinergic drugs considerably improved the jaw dystonia in three of the four patients.

The second group of patients with isolated jaw spasms described in this report present a unique syndrome with characteristic clinical and electromyographic features. Hemimasticatory spasm with facial hemiatrophy has been the subject of numerous reports. ${ }^{1011}$ The present report illustrates examples of hemimasticatory spasm occurring both with and without facial hemiatrophy. Clinically the spasms were similar in both. They were often painful, the tongue was frequently bitten by the forceful jaw closure while the spasms were precipitated by voluntary jaw closure and inhibited by jaw opening. Electromyographically, spontaneous repetitive short bursts of motor unit discharges and longer spasms were recorded from the masseter and the masseter reflexes were abnormal in both cases. The similarity of these findings to hemifacial spasm and neuromyotonia has previously been noted. ${ }^{1012}$ These features suggest that a peripheral trigeminal nerve lesion is responsible for the spasms in this condition although conclusive proof of this is lacking.

In one patient (case 7) with paroxysmal episodes of unilateral facial and masticatory spasm a diagnosis of multiple sclerosis was suspected. Although it was not possible to establish a definite diagnosis, the stereotyped nature of the attacks and the monocular adduction spasm (of case 2 of Matthews, ref 15) resembled the clinical features of paroxysmal events in multiple sclerosis described by previous authors. ${ }^{1516}$ The complex facial spasms and abnormal foot posture when walking observed in this case were also similar to the descriptions paroxysmal dystonia in multiple sclerosis. ${ }^{17}$

Involvement of the cranial musculature in tetany is unusual, but is well illustrated by case 8 . The presence of spasms involving the limb musculature and their prompt resolution after intravenous calcium distinguishes this case from the other causes of jaw spasms.
The authors thank Dr NP Leigh, Consultant neurologist, Southampton General Hospital for referring case 1 and Dr WM Carroll Consultant neurologist, Sir Charles Gairdner Hospital, Perth, Western Australia, for permission to report case 6.

\section{References}

${ }^{1}$ Gowers WR. Manual of Diseases of the Nervous System. 3rd ed, Vol 2. London: J and A Churchill, 1899:204-8.

${ }^{2}$ Marsden CD. Blepharospasm-oromandibular dystonia syndrome (Breughel's syndrome). J Neurol Neurosurg Psychiatry 1976;39:1204-9.

${ }^{3}$ Marsden CD, Tarsy D, Baldessarini RJ. Spontaneous and drug-induced movement disorders in psychotic patients. In: Benson DF, Blumer D, eds. Psychiatric Aspects of Neurologic Disease. New York, Grune and Stratton, 1975:219-66.

${ }^{4}$ Burke RE, Fahn S, Jankovic J, et al. Tardive dystonia: Late-onset and persistent dystonia caused by antipsychotic drugs. Neurology (NY) 1982;32:1335-46.

5 Von Economo C. Encephalitis Lethargica. Its Sequelae and Treatment. London: Oxford University Press, 1931:50.

${ }^{6}$ Jelasic F, Freitag V. Inverse activity of the masticatory muscles with and without trismus: a brainstem syndrome. J Neurol Neurosurg Psychiatry 1978;61: 798-804.

${ }^{7}$ Rugh JD, Solberg WK. Psychological implications in temporomandibular pain and dysfunction. Oral Sci Rev 1976;1:3-30.

${ }^{8}$ Jenkyn LR, Walsh DB, Culver CM, et al. Clinical signs in diffuse cerebral dysfunction. $J$ Neurol Neurosurg Psychiatry 1977;61:798-804.

${ }^{9}$ Satayoshi E. A syndrome of progressive muscle spasm, alopecia and diarrhoea. Neurology (Minneap) 1978; 28:458-71.

${ }^{10}$ Kaufman MD. Masticatory spasm in facial hemiatrophy. Ann Neurol 1980;7:585-7.

${ }^{11}$ Lapresle J, Desi M. Scleroderma with progressive crossed atrophy involving the left side of the body and the face. Rev Neurol (Paris) 1982;138:815-25.

12 Thompson PD, Carroll WM. Hemimasticatory spasm: a peripheral paroxysmal cranial neuropathy? J Neurol Neurosurg Psychiatry 1983;46:274-6.

${ }^{13}$ Berardelli A, Rothwell JC, Day BL, Marsden CD. Pathophysiology of Blepharospasm and Oromandibular Dystonia. Brain 1985;108:593-608.

${ }^{14}$ Mayo J, Berciano J. Cephalic tetanus presenting with Bell's palsy. J Neurol Neurosurg Psychiatry 1985; 48:290.

${ }^{15}$ Matthews WB. Paroxysmal symptoms in multiple sclerosis. J Neurol Neurosurg Psychiatry 1975;38:617-23.

${ }^{16}$ Espir MLE, Millac P. Treatment of paroxysmal disorders in multiple sclerosis with carbamazepine. J Neurol Neurosurg Psychiatry 1970;33:528-31.

${ }^{17}$ Berger JR, Sheremata WA, Melamed MD. Paroxysmal dystonia as the initial manifestation of multiple sclerosis. Arch Neurol 1984;41:747-50. 